\title{
Global rigidity for totally nonsymplectic Anosov $\mathbb{Z}^{k}$ actions
}

\author{
BORIS KALININ \\ VICTORIA SADOVSKAYA
}

\begin{abstract}
We consider a totally nonsymplectic (TNS) Anosov action of $\mathbb{Z}^{k}$ which is either uniformly quasiconformal or pinched on each coarse Lyapunov distribution. We show that such an action on a torus is $C^{\infty}$-conjugate to an action by affine automorphisms. We also obtain similar global rigidity results for actions on an arbitrary compact manifold assuming that the coarse Lyapunov foliations are topologically jointly integrable.
\end{abstract}

37C15, 37D99

\section{Introduction}

In this paper we consider Anosov actions of $\mathbb{Z}^{k}, k \geq 2$, on a compact smooth manifold. For certain classes of these actions, we establish global rigidity, ie, the existence of a smooth conjugacy to an algebraic model.

The classification of Anosov systems is one of the central problems in smooth dynamics. A long standing conjecture on topological classification is that any Anosov diffeomorphism is topologically conjugate to a hyperbolic automorphism of a torus or, more generally, an infranilmanifold. This was established under the assumption that the underlying manifold is an infranilmanifold, or that the diffeomorphism is of codimension one (Franks [5], Manning [16], Newhouse [17]). A smooth classification of Anosov diffeomorphisms is not feasible. Indeed, even when two Anosov diffeomorphisms are topologically conjugate, the conjugacy is typically only Hölder continuous.

In contrast to a single Anosov diffeomorphism, it may be possible to classify higher rank Anosov actions up to a smooth conjugacy. These are the actions of higher rank groups with at least one element acting normally hyperbolically to the orbit foliation. The study of rigidity for these actions originated from Zimmer's conjecture that the standard action of $S L(n, \mathbb{Z})$ on $\mathbb{T}^{n}, n>2$, is locally rigid, ie, any $C^{1}$-small perturbation is smoothly conjugate to the original action. The smoothness of the conjugacy was established using the action of a diagonalizable subgroup isomorphic to $\mathbb{Z}^{n-1}$ (Hurder 
[8], Katok and Lewis [12]). This prompted the study of higher rank abelian Anosov actions.

Reducible abelian actions can be obtained as products of Anosov diffeomorphisms or flows. Important irreducible examples arise from natural algebraic constructions. They include $\mathbb{Z}^{k}$ actions by automorphisms of tori and infranilmanifolds, and $\mathbb{R}^{k}$ actions by left translations on homogeneous spaces and biquotients. These actions exhibit strong rigidity phenomena such as scarcity of invariant measures and cocycle rigidity (see surveys in Lindenstrauss [15] and Nitica-Török [18]).

It is conjectured that all irreducible Anosov actions of $\mathbb{Z}^{k}$ and $\mathbb{R}^{k}, k \geq 2$, are smoothly conjugate to algebraic actions. This conjecture is supported by the local rigidity results of Katok and Spatzier. They showed that any $C^{1}$-small perturbation of a higher-rank algebraic Anosov action with semisimple linear part is smoothly conjugate to the original action [14]. There are few results, however, toward proving the conjecture. So far, all known results are for actions with one-dimensional coarse Lyapunov distributions. Such actions are often called Cartan actions. Coarse Lyapunov distributions are the finest nontrivial intersections of stable distributions of Anosov elements of the action. Katok and Lewis established global rigidity for actions of certain maximal sets of commuting diffeomorphisms of a torus [12]. In this case, the coarse Lyapunov distributions were one-dimensional stable distributions of some Anosov elements of the action. Recently, the first author obtained a smooth classification of certain classes of continuous and discrete actions of rank $k \geq 3$ on arbitrary manifolds in the joint work with Spatzier [11].

We consider Anosov $\mathbb{Z}^{k}$ actions with higher-dimensional coarse Lyapunov distributions under various assumptions on the relation between slow and fast expansion/contraction rates on these distributions. Our approach is different from those of [11] or Katok and Lewis [12], it is based on the study of holonomy maps and affine structures on coarse Lyapunov foliations. For actions on tori, in contrast to [12], we do not rely directly on the topological conjugacy given by the topological classification. This allows us to obtain results on an arbitrary manifold, provided that the coarse Lyapunov foliations are topologically jointly integrable. Also, we assume that the action is totally nonsymplectic (TNS), ie, any pair of coarse Lyapunov distributions is contracted by some element of the action. We note that this assumption is satisfied by the actions in [12] and by the discrete actions in [11].

We state our main results in Section 3 and prove them in Sections 4 and 5. In Section 2 we discuss the structure of $\mathbb{Z}^{k}$ Anosov actions and give necessary definitions.

The first author was supported in part by NSF grant DMS-0140513, and the second author was supported in part by NSF grant DMS-0401014. 


\section{Preliminaries}

\subsection{Lyapunov exponents for $\mathbb{Z}^{k}$ actions}

In this section we describe the Multiplicative Ergodic Theorem and related notions for $\mathbb{Z}^{k}$ actions (see Kalinin and Katok [10] for more details). Let $f$ be a diffeomorphism of a compact manifold $\mathcal{M}$ preserving an ergodic probability measure $\mu$. By Oseledec Multiplicative Ergodic Theorem, there exist finitely many numbers $\chi_{i}$ and a measurable splitting of the tangent bundle $T \mathcal{M}=\bigoplus E_{i}$ on a set of full measure such that the forward and backward Lyapunov exponents of $v \in E_{i}$ are $\chi_{i}$. This splitting is called Lyapunov decomposition.

Let $\alpha$ be a $\mathbb{Z}^{k}$ action on a compact manifold $\mathcal{M}$ preserving an ergodic probability measure $\mu$. By commutativity, the Lyapunov decompositions for individual elements of $\mathbb{Z}^{k}$ can be refined to a joint invariant splitting for the action. Thus the Multiplicative Ergodic Theorem in this case yields the following:

Proposition 2.1 There are finitely many linear functionals $\chi$ on $\mathbb{Z}^{k}$, a set of full measure $\mathcal{P}$ and a splitting of the tangent bundle $T \mathcal{M}=\bigoplus E_{\chi}$ over $\mathcal{P}$ which is $\alpha-$ invariant and measurable such that, for all $a \in \mathbb{Z}^{k}$ and $v \in E_{\chi}$, the Lyapunov exponent of $v$ is $\chi(a)$, ie,

$$
\lim _{n \rightarrow \pm \infty} \frac{1}{n} \log \left\|d a^{n}(v)\right\|=\chi(a),
$$

where $\|\cdot\|$ is a continuous norm on $T \mathcal{M}$.

The splitting $\bigoplus E_{\chi}$ is called the Lyapunov decomposition and the linear functionals $\chi$ are called the Lyapunov exponents or Lyapunov functionals of $\alpha$. The hyperplanes ker $\chi \subset \mathbb{R}^{k}$ are called the Lyapunov hyperplanes or Weyl chamber walls and the connected components of $\mathbb{R}^{k}-\cup_{\chi}$ ker $\chi$ are called the Weyl chambers of $\alpha$. The elements in the union of the Lyapunov hyperplanes are called singular, and the elements in the union of the Weyl chambers are called regular.

Consider a $\mathbb{Z}^{k}$ action by automorphisms, of a torus or an infranilmanifold. Then the Lyapunov decomposition is determined by the eigenspaces of the automorphisms and the Lyapunov exponents are the logarithms of the moduli of the eigenvalues. Hence they are independent of the invariant measure, the Lyapunov decomposition is smooth, and the Lyapunov functionals give uniform estimates of expansion and contraction rates. 


\subsection{Anosov $\mathbb{Z}^{k}$ actions and coarse Lyapunov distributions}

Let $f$ be a diffeomorphism of a compact manifold $\mathcal{M}$. Recall that $f$ is Anosov if there exist a continuous $f$-invariant decomposition of the tangent bundle $T \mathcal{M}=E^{s} \oplus E^{u}$ and constants $K>0, \lambda>0$ such that, for all $n \in \mathbb{N}$,

$$
\begin{aligned}
\left\|d f^{n}(v)\right\| & \leq K e^{-\lambda n}\|v\| \text { for all } v \in E^{s}, \\
\left\|d f^{-n}(v)\right\| & \leq K e^{-\lambda n}\|v\| \text { for all } v \in E^{u} .
\end{aligned}
$$

The distributions $E^{s}$ and $E^{u}$ are called the stable and unstable distributions of $f$. It is well-known that these distributions are tangential to the stable and unstable foliations $W^{s}$ and $W^{u}$ respectively. The leaves of these foliations are $C^{\infty}$ injectively immersed Euclidean spaces. Locally, the immersions vary continuously in $C^{\infty}$ topology. Such foliations are said to have uniformly $C^{\infty}$ leaves. In general, the distributions $E^{s}$ and $E^{u}$ are only Hölder continuous transversally to the corresponding foliations.

Now we consider a $\mathbb{Z}^{k}$ action $\alpha$ on a compact manifold $\mathcal{M}$. The action is called Anosov if there is an element which acts as an Anosov diffeomorphism. Note that the existence of one Anosov element implies that all Lyapunov functionals are nonzero. Hence if such an action is algebraic, ie, by automorphisms of a torus or an infranilmanifold, then all regular elements are Anosov. In general, however, it is not known whether all regular elements of an Anosov $\mathbb{Z}^{k}$ action are Anosov. To obtain a good structural theory for the general actions one needs to assume the existence of several Anosov elements.

The stable distribution of one Anosov element is invariant under any other element, and it is natural to consider intersections of the stable distributions for various Anosov elements of the action. The finest such intersections are called coarse Lyapunov distributions. This notion proved to be very useful for both algebraic and nonalgebraic actions.

For an algebraic action, the coarse Lyapunov distributions are defined everywhere and smooth. Moreover, a coarse Lyapunov distribution $E^{\chi}$ can be equivalently defined as a direct sum of all Lyapunov spaces with Lyapunov functionals positively proportional to $\chi$ :

$$
E^{\chi}=\oplus E_{\chi^{\prime}}, \quad \chi^{\prime}=c \chi \text { with } c>0 .
$$

For nonalgebraic actions, however, the situation is more complicated. It is not clear that the an intersection of several stable distributions has constant dimension. Also, the distribution $\oplus E_{\chi^{\prime}}$ in general is only measurable and defined almost everywhere. The next proposition shows that, in the presence of sufficiently many Anosov elements, the 
coarse Lyapunov distributions are well-defined, continuous, and tangent to foliations with smooth leaves. This is the discrete version of Proposition 2.4 in [11]. We denote the set of all Anosov elements in $\mathbb{Z}^{k}$ by $\mathcal{A}$.

Proposition 2.2 Let $\alpha$ be an Anosov $\mathbb{Z}^{k}$-action and let $\mu$ be an ergodic probability measure for $\alpha$ with full support. Suppose that there exits an Anosov element in every Weyl chamber defined by $\mu$. Then for each Lyapunov exponent $\chi$ the coarse Lyapunov distribution can be defined as

$$
E^{\chi}(p)=\bigcap_{\{a \in \mathcal{A} \mid \chi(a)<0\}} E_{a}^{s}(p)=\bigoplus_{\left\{\chi^{\prime}=c \chi \mid c>0\right\}} E_{\chi^{\prime}}(p)
$$

on the set $\mathcal{P}$ of full measure where the Lyapunov exponents exist. Moreover, $E^{\chi}$ is Hölder continuous, and thus it can be extended to a Hölder distribution tangent to the foliation $W^{\chi}=\bigcap_{\{a \in \mathcal{A} \mid \chi(a)<0\}} W_{a}^{s}$ with uniformly $C^{\infty}$ leaves.

It is easy to see that the coarse Lyapunov distributions constructed in the proposition are indeed the finest nontrivial intersections of various stable distributions. Extending Proposition 2.9 in [11], one can also show that for any other invariant measure with Anosov elements in every Weyl chamber, (2-1) gives the same distributions. Note that ergodic measures with full support always exist if the action contains a transitive Anosov element. A natural example is given by the measure of maximal entropy for such an element which, by uniqueness, is invariant under the action.

Now we describe an important class of $\mathbb{Z}^{k}$ actions called totally nonsymplectic, or TNS. For such an action, any pair of coarse Lyapunov distributions is contracted by some element. For an algebraic action this is equivalent to having no negatively proportional Lyapunov functionals. This property proved important and motivated the definition for nonalgebraic actions in Katok-Niţică-Török [13] similar to the following:

Definition 2.3 Let $\alpha$ be a $C^{\infty}$ action of $\mathbb{Z}^{k}$ with a transitive Anosov element on a compact smooth connected manifold $\mathcal{M}$. We say that $\alpha$ is a TNS Anosov action if $T \mathcal{M}$ splits into a direct sum of continuous distributions $E^{i}$, called the coarse Lyapunov distributions, so that:

(1) Any distribution $E^{i}$ is of the form $E^{i}=\bigcap_{a \in \mathcal{A}} E_{a}^{\sigma(a)}$, where $\mathcal{A}$ is the set of Anosov elements and $\sigma(a)$ is either s or $\mathrm{u}$.

(2) The sum of any two distributions $E^{i}$ and $E^{j}$ is contained in $E_{a}^{s}$ for some $a \in \mathcal{A}$.

Remark 2.4 Note that condition (1) ensures that the distributions $E^{i}$ are exactly the coarse Lyapunov distributions as described earlier. It also implies that the distributions 
$E^{i}$ are Hölder continuous and tangent to the integral foliations $W^{i}$ with uniformly $C^{\infty}$ leaves, called the coarse Lyapunov foliations.

It also follows that for any $E^{i} \neq E^{j}$ there exists $a \in \mathcal{A}$ for which $E^{i} \subset E_{a}^{s}$ and $E^{j} \subset E_{a}^{u}$.

This definition of an Anosov TNS action is very similar to the one introduced in [13]. In that definition, the set of all Anosov elements was replaced by a subcollection. It gives similar properties for the action, but the distributions $E^{i}$ may turn out to be larger than the actual coarse Lyapunov distributions. We consider such distributions in Theorem 3.4.

Proposition 2.2 gives the following sufficient conditions for an action to be TNS Anosov. These conditions are close to being necessary.

Corollary 2.5 Let $\alpha$ be a $C^{\infty}$ action of $\mathbb{Z}^{k}$ with a transitive Anosov element on a compact smooth connected manifold $\mathcal{M}$. Suppose that for some $\alpha$-invariant ergodic measure $\mu$ with full support there are no negatively proportional Lyapunov functionals, and in each Weyl chamber there exists an Anosov element. Then $\alpha$ is a TNS Anosov action.

\subsection{Conjugacy to algebraic models}

Let $f$ be an Anosov diffeomorphism of a torus or, more generally, an infranilmanifold $\mathcal{N}$. By the results of Franks [5] and Manning [16], $f$ is topologically conjugate to an Anosov automorphism $A: \mathcal{N} \rightarrow \mathcal{N}$, ie, there exists a homeomorphism $\phi: \mathcal{N} \rightarrow \mathcal{N}$ such that $A \circ \phi=\phi \circ f$. The conjugacy $\phi$ is unique in the homotopy class of identity.

Now consider an Anosov $\mathbb{Z}^{k}$ action $\alpha$ on an infranilmanifold. For any Anosov element of $\alpha$ there is a homeomorphism $\phi$ which conjugates it to an automorphism. It is well known that $\phi$ then conjugates $\alpha$ to an action by affine automorphisms, Hurder [8, proof of Proposition 2.18]. This follows from the fact that any homeomorphism commuting with an Anosov automorphism is an affine automorphism (Palis and Yoccoz [19, proof of Proposition 0]). By an affine automorphism we mean a composition of an automorphism and a translation. We note that an Anosov $\mathbb{Z}^{k}$ action on an infranilmanifold may have no fixed points [8], however, there is always a finite index subgroup which fixes a point and whose action is conjugate to an action by automorphisms.

\subsection{Joint integrability}

While any coarse Lyapunov distribution is integrable, a sum of two coarse Lyapunov distributions is not integrable in general. This can be observed for $\mathbb{Z}^{k}$ actions on 
nilmanifolds. However, for an algebraic $\mathbb{Z}^{k}$ action on a torus, all Lyapunov foliations are linear, and thus the sum of any two coarse Lyapunov distributions is integrable.

Let $\alpha$ be an Anosov $\mathbb{Z}^{k}$ action on a torus. Then it is topologically conjugate to an algebraic action (Section 2.3). The conjugacy maps the (un)stable foliations to the (un)stable foliations, and hence it maps the coarse Lyapunov foliations to the coarse Lyapunov foliations. We conclude that any two coarse Lyapunov foliations for $\alpha$ are topologically jointly integrable in the following sense.

Definition 2.6 Two foliations $W^{1}$ and $W^{2}$ of a manifold are topologically jointly integrable if there is a topological foliation $W$ such that for any $x$ the map

$$
\phi: W^{1}(x) \times W^{2}(x) \rightarrow W(x), \quad \phi(y, z)=W^{1}(z) \cap W^{2}(y)
$$

is a well-defined local homeomorphism. In other words, the foliations $W^{1}$ and $W^{2}$ give a local product structure on the leaves of $W$.

\subsection{Conformality and uniform quasiconformality}

Let $f$ be a diffeomorphism of a compact Riemannian manifold $\mathcal{M}$, and let $E$ be a continuous $f$-invariant distribution. The diffeomorphism $f$ is uniformly quasiconformal on $E$ if the quasiconformal distortion

$$
K_{E}(x, n)=\frac{\max \left\{\left\|d f^{n}(v)\right\|: v \in E(x),\|v\|=1\right\}}{\min \left\{\left\|d f^{n}(v)\right\|: v \in E(x),\|v\|=1\right\}}
$$

is uniformly bounded for all $n \in \mathbb{Z}$ and $x \in \mathcal{M}$. We note that the notion of uniform quasiconformality does not depend on the choice of a Riemannian metric on the manifold. Clearly, an Anosov diffeomorphism can be uniformly quasiconformal on $E$ only if $E$ is contained in its stable or its unstable distribution.

If $K_{E}(x, n)=1$ for all $x$ and $n$, the diffeomorphism is said to be conformal on $E$. The next result shows that any Anosov diffeomorphism uniformly quasiconformal on $E$ is, in fact, conformal with respect to some metric on $E$.

Theorem 2.7 Let $f$ be a topologically transitive $C^{\infty}$ Anosov diffeomorphism on a compact manifold $\mathcal{M}$. Let $E$ be a Hölder continuous $f$-invariant distribution. Suppose that $f$ is uniformly quasiconformal on $E$. Then $f$ is conformal with respect to a Riemannian metric on $E$ which is Hölder continuous on $\mathcal{M}$.

If, in addition, $E$ is tangential to a foliation $W$ with $C^{\infty}$ leaves, then this metric is $C^{\infty}$ along the leaves of $W$. 
The proof of this theorem is virtually identical to the proof of Theorem 1.3 in Sadovskaya [21]. There the stable distribution and the stable foliation are considered instead of $E$ and $W$.

Let $\Gamma$ be a group acting on $\mathcal{M}$ via diffeomorphisms, and let $E$ be a continuous $\Gamma$-invariant distribution. We say that the action is uniformly quasiconformal on $E$ if the quasiconformal distortion is uniformly bounded for all elements of $\Gamma$.

Proposition 2.8 Suppose that the $\Gamma$-action is generated by finitely many commuting diffeomorphisms. If each generator is uniformly quasiconformal on $E$, then the $\Gamma-$ action is uniformly quasiconformal on $E$.

Proof Any element of the action can be written as $f_{1}^{n_{1}} \ldots f_{k}^{n_{k}}$, where $f_{1}, \ldots, f_{k}$ are the generators. Then uniform quasiconformality of the action follows directly from the definition.

\section{Main results}

In our first theorem we consider uniformly quasiconformal TNS Anosov actions.

Theorem 3.1 Let $\alpha$ be a TNS Anosov action of $\mathbb{Z}^{k}$ on a compact connected smooth manifold $\mathcal{M}$. Suppose that:

(1) Any two coarse Lyapunov foliations are topologically jointly integrable.

(2) The action is uniformly quasiconformal on each coarse Lyapunov distribution.

Then a finite cover of $\alpha$ is $C^{\infty}$-conjugate to a $\mathbb{Z}^{k}$ action by affine automorphisms of a torus.

If the manifold $\mathcal{M}$ is a torus, then condition (1) is automatically satisfied (see Section 2.4). Thus we obtain the following corollary.

Corollary 3.2 Let $\alpha$ be a TNS Anosov action of $\mathbb{Z}^{k}$ on a torus. Suppose that the action is uniformly quasiconformal on each coarse Lyapunov distribution. Then $\alpha$ is $C^{\infty}$-conjugate to a $\mathbb{Z}^{k}$ action by affine automorphisms of the torus.

Note that some or all coarse Lyapunov distributions may be one dimensional. In this case, the quasiconformality assumption is trivially satisfied. In higher dimensions, quasiconformality can be replaced by certain pinching, ie, a relationship between the slow and fast rates of expansion/contraction. The following theorem gives a result of this type. Note, however, that uniform quasiconformality does not relate the expansion/contraction rates at different points, so it does not imply the 1/2-pinching below. 
Definition 3.3 Let $f$ a diffeomorphism which contracts an invariant distribution $E$. We say that $f$ is $1 / 2-$ pinched on $E$ if there exist numbers $K>0$ and $0<\mu<\lambda$ with $\lambda<2 \mu$ such that for any $v \in E$,

$$
K^{-1} e^{-n \lambda}\|v\| \leq\left\|d f^{n}(v)\right\| \leq K e^{-n \mu}\|v\| .
$$

Theorem 3.4 Let $\alpha$ be an Anosov action of $\mathbb{Z}^{k}$ on a compact connected smooth manifold $\mathcal{M}$. Suppose that $T \mathcal{M}$ splits into a direct sum of continuous distributions $E^{i}$, where each $E^{i}$ is an intersection of stable distributions of some Anosov elements of the action, and that for any two distributions $E^{i}$ and $E^{j}$ :

(1) The corresponding foliations are topologically jointly integrable.

(2) There is an element which contracts both distributions and is 1/2-pinched on $E^{i}$.

(3) There is an element $a \in \mathbb{Z}^{k}$ which expands $E^{i}$ faster than it expands $E^{j}$, ie, for any $x \in \mathcal{M}$,

$$
\max \left\{\|d a(v)\|: v \in E^{j}(x),\|v\|=1\right\}<\min \left\{\|d a(v)\|: v \in E^{i}(x),\|v\|=1\right\} .
$$

Then $\alpha$ is $C^{\infty}$-conjugate to a $\mathbb{Z}^{k}$ action by affine automorphisms of an infranilmanifold.

Applying this theorem to actions on tori, we obtain the following result.

Corollary 3.5 Let $\alpha$ be an Anosov action of $\mathbb{Z}^{k}$ on a torus satisfying conditions (2) and (3) of Theorem 3.4. Then $\alpha$ is $C^{\infty}$-conjugate to a $\mathbb{Z}^{k}$ action by affine automorphisms of the torus.

Remark 3.6 Condition (3) is used in Theorem 3.4 only to obtain certain smoothness of $E^{i}$ along the leaves of the foliation $W^{j}$ tangential to $E^{j}$. It can be substituted by the assumption that the regularity of $E^{i}$ along $W^{j}$ is $C^{1, \beta}$ for all $\beta<1$.

In Theorem 3.4, each distribution $E^{i}$ may be a direct sum of several coarse Lyapunov distributions (see the discussion after Remark 2.4). If the distributions $E^{i}$ are the coarse Lyapunov distributions, then the infranilmanifold is finitely covered by a torus.

Corollary 3.7 Let $\alpha$ be a TNS Anosov action of $\mathbb{Z}^{k}$ on a compact connected smooth manifold $\mathcal{M}$. Suppose that the coarse Lyapunov distributions satisfy conditions (1), (2), and (3) of Theorem 3.4. Then a finite cover of $\alpha$ is $C^{\infty}$-conjugate to a $\mathbb{Z}^{k}$ action by affine automorphisms of a torus. 
We can also deduce some results on local rigidity of algebraic Anosov actions. The local rigidity of higher rank Anosov $\mathbb{Z}^{k}$ actions by automorphisms of tori was proved by Katok and Spatzier [14] under the assumption that the automorphisms are semisimple (ie, have no Jordan blocks). The general case has not been resolved. In a recent preprint [2], Einsiedler and Fisher considered the Jordan block case under the 1/2-pinching assumption on each coarse Lyapunov distribution. Corollary 3.8 gives an alternative proof of their result in the TNS case.

Corollary 3.8 Let $\alpha$ be a TNS Anosov action of $\mathbb{Z}^{k}$ by toral automorphisms. Suppose that for each coarse Lyapunov distribution there exists a regular element which is 1/2-pinched on this distribution.

Then $\alpha$ is locally rigid, ie, it is $C^{\infty}$-conjugate to any sufficiently $C^{1}$-small perturbation.

More generally, Corollary 3.7 can be applied to actions which have pinching similar to that of small perturbations of 1/2-pinched algebraic actions.

Corollary 3.9 Let $\alpha$ be a TNS Anosov action of $\mathbb{Z}^{k}$ on a compact connected smooth manifold $\mathcal{M}$. Suppose that:

(1) Any two coarse Lyapunov foliations are topologically jointly integrable.

(2) For any coarse Lyapunov distribution $E$ there exist functionals $\chi_{\min }$ and $\chi_{\max }$ proportional with a constant $1 \leq c<2$, such that for some $K>0$

$$
K^{-1} e^{\chi_{\min }(a)}\|v\| \leq\|d a(v)\| \leq K e^{\chi_{\max }(a)}\|v\|
$$

for any $a \in \mathbb{Z}^{k}$ with $\chi_{\min }(a)>0$ and any $v \in E$.

Then a finite cover of $\alpha$ is $C^{\infty}$-conjugate to a $\mathbb{Z}^{k}$ action by affine automorphisms of a torus.

Remark 3.10 The inequality in condition (2) of Corollary 3.9 of can be replaced by a weaker assumption

$$
K^{-1} e^{\chi_{\min }(a)-\varepsilon|a|}\|v\| \leq\|d a(v)\| \leq K e^{\chi_{\max }(a)+\varepsilon|a|}\|v\|
$$

if $\varepsilon$ is small enough for the given system of functionals. 


\section{Proof of Theorem 3.1}

\subsection{Outline of the proof}

We begin by considering any two coarse Lyapunov distributions $E^{1}$ and $E^{2}$, and the corresponding coarse Lyapunov foliations $W^{1}$ and $W^{2}$. In Sections 4.2 and 4.3 we show that the leaves of $W=W^{1} \oplus W^{2}$ are smooth, and that $E^{1}$ and $E^{2}$ are $C^{1, \beta}$ along these leaves.

In Section 4.4 we discuss the nonstationary linearization of the action along the leaves of $W^{1}$ and the associated affine structures on these leaves. In the next section we show that the holonomies of the foliation $W^{2}$ between the leaves of $W^{1}$ are affine. This implies that $E^{2}$ is $C^{\infty}$ along $W^{1}$. It follows that all coarse Lyapunov distributions are $C^{\infty}$ on $\mathcal{M}$.

In Section 4.6 we show that the nonstationary linearizations depend smoothly on the base point. We use this to construct a $C^{\infty}$ invariant affine connection in Section 4.7. Together with the smoothness of the coarse Lyapunov distributions, this implies that the action is $C^{\infty}$-conjugate to an action by affine automorphisms of an infranilmanifold. Finally, we show that this infranilmanifold is finitely covered by a torus.

\subsection{Regularity of the sum of two coarse Lyapunov foliations}

Since $\alpha$ is a TNS Anosov action, the coarse Lyapunov distributions are defined everywhere on the manifold and Hölder continuous. They are tangential to coarse Lyapunov foliations with uniformly $C^{\infty}$ leaves. (See Section 2.2.)

We consider any two coarse Lyapunov distributions $E^{1}$ and $E^{2}$, and the corresponding coarse Lyapunov foliations $W^{1}$ and $W^{2}$. By assumption (1) of the theorem, the foliations $W^{1}$ and $W^{2}$ are topologically jointly integrable, see Definition 2.6. We will write $W^{1} \oplus W^{2}$ for the foliation $W$ in the definition. Since the action is TNS, there is an element which contracts $W$. Applying the iterates of its inverse, one can see that the local product structure on the leaves of $W$ extends to the global one.

Lemma 4.1 The topological foliation $W=W^{1} \oplus W^{2}$ has uniformly $C^{\infty}$ leaves tangent to the distribution $E=E^{1} \oplus E^{2}$.

Proof This follows from the Journé lemma below. A function is said to be uniformly $C^{\infty}$ along a foliation with smooth leaves if the partial derivatives of all orders in the foliation directions exist and are continuous on the manifold. 
Lemma 4.2 [9] Let $\mathcal{M}$ be a $C^{\infty}$ manifold, and let $W^{1}$ and $W^{2}$ be two transverse Hölder continuous foliations with uniformly $C^{\infty}$ leaves. If a function $\phi$ is uniformly $C^{\infty}$ along the leaves of the two foliations, then it is $C^{\infty}$ on $\mathcal{M}$.

We recall that the leaves of $W^{1}$ and $W^{2}$ are $C^{\infty}$ immersed manifolds tangent to the distributions $E^{1}$ and $E^{2}$ respectively. Moreover, they are uniformly $C^{\infty}$. We can identify a small neighborhood of a point $x \in \mathcal{M}$ with a ball in $\mathbb{R}^{n}$ and consider the orthogonal projection $\Pi$ to the subspace $E(x)=E^{1}(x) \oplus E^{2}(x)$. The restriction of $\Pi$ to the leaf $W(x)$ is locally a homeomorphism that projects foliations $W^{1}$ and $W^{2}$ to transverse foliations $\bar{W}^{1}$ and $\bar{W}^{2}$ in $E(x)$ with uniformly $C^{\infty}$ leaves. Then the inverse $\phi$ of this restriction is uniformly $C^{\infty}$ along the leaves of $\bar{W}^{1}$ and $\bar{W}^{2}$ and hence it is $C^{\infty}$ by the Journe lemma. This implies that $W(x)$ is a $C^{\infty}$ immersed manifold. In particular, its tangent distribution is $E=E^{1} \oplus E^{2}$. Since the immersions of $W^{1}(x)$ and $W^{2}(x)$ depend continuously on $x$ in $C^{\infty}$ topology it is easy to see that so do the immersions of $W(x)$.

\subsection{Coarse Lyapunov distributions are $C^{1, \beta}$ along $W$}

Let $E^{1}$ and $E^{2}$ be two coarse Lyapunov distributions, let $E=E^{1} \oplus E^{2}$ be their sum, and let $W=W^{1} \oplus W^{2}$ be the corresponding foliation. Above we established that the leaves of this foliation are $C^{\infty}$ manifolds. We study regularity of the distributions $E^{1}$ and $E^{2}$ along the leaves of $W$.

Proposition 4.3 Suppose that there exists an element $f$ that expands $E^{1}$, contracts $E^{2}$ and is uniformly quasiconformal on $W^{2}$. Then $E^{1}$ is $C^{1, \beta}$ along the leaves of $W$ for some $\beta>0$. More precisely, its first derivatives in $W$ directions exist and are Hölder continuous along the leaves of $W$ with uniform bounds on $\mathcal{M}$ for the derivatives and the Hölder constants.

Proof We use the $C^{r}$ Section Theorem of M Hirsch, C Pugh, and M Shub. See Theorem 3.2, as well as its more detailed version Theorem 3.5, and Remarks 1 and 2 after Theorem 3.8 in [7].

We consider the distribution $E=E^{1} \oplus E^{2}$. This is a continuous bundle over $\mathcal{M}$ which is $C^{\infty}$ smooth along the leaves of $W$. There exist distributions $\bar{E}^{1}$ and $\bar{E}^{2}$ in $E$ which are close to $E^{1}$ and $E^{2}$ respectively and $C^{\infty}$ along the leaves of $W$. Now we can consider a vector bundle $\mathcal{L}$ whose fiber over $x$ is the set of linear operators from $\bar{E}^{1}(x)$ to $\bar{E}^{2}(x)$. The differential of $f$ induces a natural action $F$ on $\mathcal{L}$. Suppose that $F$ contracts fibers of $\mathcal{L}$, ie, for any $x \in \mathcal{M}$ and any $u, v \in \mathcal{L}(x)$

$$
\|F(u)-F(v)\|_{f x} \leq k_{x}\|u-v\|_{x} \text { with } \sup _{x \in M} k_{x}<1
$$


with respect to some norm on $\mathcal{L}$. Then Theorem 3.1 in [7] gives existence, uniqueness, and continuity of the invariant bounded section. By uniqueness, the graph of this invariant section is the distribution $E^{1}$. Moreover, the $C^{r}$ Section Theorem [7, Theorems 3.2 and 3.5] states that if $\mathcal{L}$ is $C^{r}$ and

$$
\sup _{x \in M} k_{x} \alpha_{x}^{r}<1 \text { where } \alpha_{x}=\left\|\left(d f_{x}\right)^{-1}\right\|
$$

then the invariant section is $C^{r}$ smooth. Theorem 3.8 in [7] and Remarks 1 and 2 after it imply that this conclusion holds for noninteger values of $r$ in the Hölder category, and that the compactness of the base can be replaced by the boundedness condition as in Theorem 3.2 in [7].

We are interested only in the smoothness of the invariant section along the leaves of $W$. In this case, we need the smoothness of the bundle $\mathcal{L}$ only along $W$ and we can use $\alpha_{x}=\left\|\left(\left.d f\right|_{E(x)}\right)^{-1}\right\|$ in (4-1). This can be also seen by formally applying the $C^{r}$ Section Theorem to the bundle $\mathcal{L}$ over the disjoint union of the leaves of $W$ as the base.

Now we use the assumption that $f$ is uniformly quasiconformal on $W^{2}$ to verify (4-1). We fix some continuous norm on the distribution $E$ and endow the fibers of $\mathcal{L}$ with the standard operator norm. We denote

$$
\begin{aligned}
l_{x} & =\min \left\{\left\|d f_{x}(v)\right\|: v \in E^{1}(x),\|v\|=1\right\} \\
m_{x} & =\min \left\{\left\|d f_{x}(v)\right\|: v \in E^{2}(x),\|v\|=1\right\} \\
M_{x} & =\max \left\{\left\|d f_{x}(v)\right\|: v \in E^{2}(x),\|v\|=1\right\} .
\end{aligned}
$$

Then

$$
\alpha_{x}=\left\|\left(\left.d f\right|_{E(x)}\right)^{-1}\right\|=1 / m_{x} \quad \text { and } \quad k_{x} \approx M_{x} / l_{x}
$$

since $\bar{E}^{1}$ and $\bar{E}^{2}$ are close to $E^{1}$ and $E^{2}$. Hence

$$
k_{x} \alpha_{x}^{1+\beta} \approx \frac{M_{x}}{l_{x} m_{x}^{1+\beta}} \leq \frac{1}{l \cdot m_{x}^{\beta}} \cdot \frac{M_{x}}{m_{x}} \leq \frac{K}{l \cdot\left(\inf _{x}\left\{m_{x}\right\}\right)^{\beta}}
$$

where $l=\inf _{x} l_{x}$ and

$$
K=\sup _{x, n} \frac{\max \left\{\left\|d f^{n}(v)\right\|: v \in E^{2}(x),\|v\|=1\right\}}{\min \left\{\left\|d f^{n}(v)\right\|: v \in E^{2}(x),\|v\|=1\right\}}
$$

is the quasiconformal distortion bound. Note that $\mathrm{K}$ is a uniform bound for all iterates $f^{n}$. Since $f$ expands $E^{1}$ we can replace it by $f^{n}$, if necessary, to ensure that $l$ is large enough so that $K / l<1$. Then the right hand side is less than 1 for some $\beta>0$. Once this iterate is chosen, we can take $\bar{E}^{1}$ and $\bar{E}^{2}$ close enough to $E^{1}$ and 
$E^{2}$ to guarantee that $\sup _{x \in M} k_{x} \alpha_{x}^{1+\beta}<1$. Hence, by the $C^{r}$ Section Theorem, the distribution $E^{1}$ is $C^{1, \beta}$ smooth along the leaves of $W$.

By Remark 2.4, for any two coarse Lyapunov distributions there exists an element of the action which expands the first distribution and contracts the second one. Thus, under the assumptions of Theorem 3.1, Proposition 4.3 implies that any two coarse Lyapunov foliations $W^{1}$ and $W^{2}$ are $C^{1, \beta}$ smooth along the leaves of their sum.

\subsection{Nonstationary linearizations}

For each coarse Lyapunov foliation $W=W^{i}$ we use the following proposition to obtain a nonstationary linearization $h=h^{i}$ of the action along the leaves of $W$.

Proposition 4.4 [21, Proposition 4.1] Let $f$ be a diffeomorphism of a compact Riemannian manifold $\mathcal{M}$. Let $W$ be a continuous invariant foliation with $C^{\infty}$ leaves, and let $E$ be its tangent distribution.

Suppose that $\left\|\left.d f\right|_{E}\right\|<1$, and there exist $K>0$ and $\varepsilon>0$ such that for any $x \in \mathcal{M}$ and $n \in \mathbb{N}$,

$$
\left\|\left(\left.d f^{n}\right|_{E(x)}\right)^{-1}\right\| \cdot\left\|\left.d f^{n}\right|_{E(x)}\right\|^{2} \leq K(1-\varepsilon)^{n} .
$$

Then for any $x \in \mathcal{M}$ there exists a $C^{\infty}$ diffeomorphism $h_{x}: W(x) \rightarrow E(x)$ such that

(1) $h_{f x} \circ f=d f_{x} \circ h_{x}$;

(2) $h_{x}(x)=0$ and $d h_{x}(x)$ is the identity map;

(3) $h_{x}$ depends continuously on $x$ in $C^{\infty}$ topology.

Let $f$ be an element of the action which contracts a coarse Lyapunov foliation $W$. Since $f$ is uniformly quasiconformal on $W$,

$$
\left\|\left(\left.d f^{n}\right|_{E(x)}\right)^{-1}\right\| \cdot\left\|\left.d f^{n}\right|_{E(x)}\right\|
$$

is uniformly bounded in $x$ and $n$. Hence (4-2) is satisfied and there exists a linearization $h$ for $f$ along the leaves of $W$. Since such a linearization is unique [21, Lemma 4.1], $h$ linearizes any diffeomorphism which commutes with $f$. Indeed, if $g \circ f=f \circ g$, then it is easy to see that $d g^{-1} \circ h \circ g$ also gives a linearization for $f$, and hence $h \circ g=d g \circ h$. Therefore, $h$ provides linearization for all elements of the action.

Lemma 4.5 Under the assumptions of Proposition 4.4, for any $R>0$ there exists $K>0$ such that for any two points $x$ and $z$ on the same leaf of $W$ with dist $(x, z)<R$ and any $n>0$

$$
\left\|\left.d f^{n}\right|_{E(z)}\right\| \leq K \cdot\left\|\left.d f^{n}\right|_{E(x)}\right\|
$$


Proof Using the linearization $h$ along the leaves of $W$ we can write

$$
\begin{aligned}
\left.f^{n}\right|_{W(x)} & =\left.\left(h_{f^{n} x}\right)^{-1} \circ d f^{n}\right|_{E(x)} \circ h_{x}, \\
\text { so }\left.d f^{n}\right|_{E(z)} & =\left.\left(d h_{f^{n} x}\left(f^{n} z\right)\right)^{-1} \circ d f^{n}\right|_{E(x)} \circ d h_{x}(z) .
\end{aligned}
$$

Since $h_{x}$ depends continuously on $x$ in $C^{\infty}$ topology, $\left\|d h_{x}(y)\right\|$ and $\left\|\left(d h_{x}(y)\right)^{-1}\right\|$ are uniformly bounded for all $x \in \mathcal{M}$ and $y \in W(x)$ with dist $(x, y)<R$. Since $\operatorname{dist}\left(f^{n} x, f^{n} z\right)<\operatorname{dist}(x, z)<R$, the norms of the first and last term in the right hand side are uniformly bounded and the lemma follows.

Proposition 4.6 Under the assumptions of Proposition 4.4, the map

$$
h_{z} \circ h_{x}^{-1}: E(x) \rightarrow E(z)
$$

is affine for any $x$ and $z$ on the same leaf of $W$. Hence the nonstationary linearization $h$ defines affine structures on the leaves of $W$.

By an affine structure we understand an atlas with affine transition maps.

Proof It suffices to show that

$$
d\left(h_{z} \circ h_{x}^{-1}\right)(\bar{y})=d\left(h_{z} \circ h_{x}^{-1}\right)\left(0_{x}\right) \text { for any } \bar{y} \in E(x) .
$$

Since $h_{x}=\left.\left(\left.d f^{n}\right|_{E(x)}\right)^{-1} \circ h_{f^{n} x} \circ f^{n}\right|_{W(x)}$ we obtain

$$
h_{z} \circ h_{x}^{-1}=\left.\left(\left.d f^{n}\right|_{E(z)}\right)^{-1} \circ h_{f^{n} z} \circ\left(h_{f_{n} x}\right)^{-1} \circ d f^{n}\right|_{E(x)} .
$$

Then for any $\bar{y} \in E(x)$

$$
d\left(h_{z} \circ h_{x}^{-1}\right)(\bar{y})=\left.\left(\left.d f^{n}\right|_{E(z)}\right)^{-1} \circ d h_{f^{n} z}\left(f^{n} y\right) \circ\left(d h_{f_{n} x}\left(f^{n} y\right)\right)^{-1} \circ d f^{n}\right|_{E(x)},
$$

where $y=\left(h_{x}\right)^{-1}(\bar{y}) \in W(x)$. Hence

$$
\begin{aligned}
& \left\|d\left(h_{z} \circ h_{x}^{-1}\right)(\bar{y})-d\left(h_{z} \circ h_{x}^{-1}\right)\left(0_{x}\right)\right\| \leq \\
& \left\|\left(\left.d f^{n}\right|_{E(z)}\right)^{-1}\right\| \cdot \| d h_{f^{n} z}\left(f^{n} y\right) \circ\left(d h_{f_{n} x}\left(f^{n} y\right)\right)^{-1}- \\
& \left.d h_{f^{n} z}\left(f^{n} x\right) \circ\left(d h_{f_{n} x}\left(f^{n} x\right)\right)^{-1}\|\cdot\| d f^{n}\right|_{E(x)} \| .
\end{aligned}
$$

Note that all four differentials of $h$ in the middle term are close to the identity Id (in fact $\left.d h_{f_{n} x}\left(f^{n} x\right)=\mathrm{Id}\right)$. More precisely, $d h_{x}(y)$ depends Lipschitz continuously on $y \in W(x)$ with dist $(x, y)<R$ and the Lipschitz constant is uniform in $x \in \mathcal{M}$. Hence the norm of the difference between each of these four differentials and Id is of order

$$
\max \left\{\operatorname{dist}\left(f^{n} x, f^{n} y\right), \operatorname{dist}\left(f^{n} y, f^{n} z\right)\right\} \leq K_{1}\left\|\left.d f^{n}\right|_{E(z)}\right\|,
$$


as easily follows from Lemma 4.5. Applying this lemma again we obtain

$$
\begin{aligned}
& \left\|d\left(h_{z} \circ h_{x}^{-1}\right)(\bar{y})-d\left(h_{z} \circ h_{x}^{-1}\right)\left(0_{x}\right)\right\| \leq \\
& \quad\left\|\left(\left.d f^{n}\right|_{E(z)}\right)^{-1}\right\| \cdot K_{2}\left\|\left.d f^{n}\right|_{E(z)}\right\| \cdot K\left\|\left.d f^{n}\right|_{E(z)}\right\| \rightarrow 0
\end{aligned}
$$

by (4-2). This shows that the differential of $h_{z} \circ h_{x}^{-1}$ is constant on $E(x)$ and thus the map is affine.

We conclude that for every coarse Lyapunov foliation there exist affine structures on its leaves. The maps induced by the elements of the action on the leaves are affine with respect to these structures.

\subsection{Holonomies are affine and coarse Lyapunov distributions are $C^{\infty}$}

We continue to study the regularity of two coarse Lyapunov foliations $W^{1}$ and $W^{2}$ along the leaves of their sum $W=W^{1} \oplus W^{2}$. In this section we consider holonomy maps $H$ between the leaves of $W^{1}$ along the leaves of $W^{2}$. Let $x$ and $z$ be two nearby points on the same leaf of $W^{2}$. For a point $y$ in $W^{1}(x)$ close to $x$, we denote

$$
H(y)=H_{x z}(y)=W^{2}(y) \cap W^{1}(z) .
$$

Our goal is to show that the holonomies are affine with respect to the affine structures on the leaves of $W^{1}$.

Proposition 4.7 Let $f$ be a diffeomorphism which contracts $W^{1} \oplus W^{2}$. Suppose that there exists $0<\beta \leq 1$ such that the holonomy maps are $C^{1, \beta}$ and for any two nearby points $x$ and $z$ on the same leaf of $W^{2}$,

$$
\left\|\left(\left.d f^{n}\right|_{E^{1}(z)}\right)^{-1}\right\| \cdot\left\|\left.d f^{n}\right|_{E^{1}(x)}\right\|^{1+\beta} \rightarrow 0 \quad \text { as } n \rightarrow \infty .
$$

Then the holonomy maps are affine with respect to the affine structures on the leaves of $W^{1}$ and are uniformly $C^{\infty}$, ie, they depend continuously on $x$ and $z$ in $C^{\infty}$ topology. Also, the distribution $E^{2}$ is uniformly $C^{\infty}$ along the leaves of $W$.

Proof Since the diffeomorphism $f$ contracts both $W^{1}$ and $W^{2}$, the holonomy map $H=H_{x z}: W^{1}(x) \rightarrow W^{1}(z)$ is defined on the whole leaf $W^{1}(x)$. Indeed, for any $y$

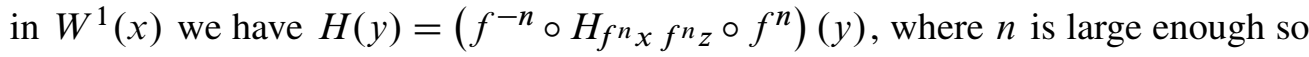
that the points $f^{n} x, f^{n} y$, and $f^{n} z$ are close.

Let $h_{x}: W^{1}(x) \rightarrow E^{1}(x)$ and $h_{z}: W^{1}(z) \rightarrow E^{1}(z)$ be the linearizations given by Proposition 4.4. Consider the map

$$
\bar{H}=\bar{H}_{x z}=h_{z} \circ H \circ\left(h_{x}\right)^{-1}: E^{1}(x) \rightarrow E^{1}(z) .
$$


Our goal is to show that $\bar{H}$ is linear and hence $H$ is affine. Note that $\bar{H}\left(0_{x}\right)=0_{z}$ since $h_{x}(x)=0_{x}$ and $h_{z}(z)=0_{z}$. For a point $y$ in $W^{1}(x)$, we denote $\bar{y}=h_{x}(y)$. To prove that the map $\bar{H}$ is linear it suffices to show that $d \bar{H}(\bar{y})=d \bar{H}\left(0_{x}\right)$ for any $y$ in $W^{1}(x)$.

Using forward iterations of the diffeomorphism $f$, we can write

$$
\bar{H}=\left.\left(\left.d f^{n}\right|_{E^{1}(z)}\right)^{-1} \circ \bar{H}_{n} \circ d f^{n}\right|_{E^{1}(x)},
$$

where

$$
\bar{H}_{n}=h_{f^{n} z} \circ H_{n} \circ\left(h_{f^{n} x}\right)^{-1}: E^{1}\left(f^{n} x\right) \rightarrow E^{1}\left(f^{n} z\right),
$$

and $H_{n}: W^{1}\left(f^{n} x\right) \rightarrow W^{1}\left(f^{n} z\right)$ is the holonomy map along the leaves of $W^{2}$. Then

$$
d \bar{H}=\left.\left(\left.d f^{n}\right|_{E^{1}(z)}\right)^{-1} \circ d \bar{H}_{n} \circ d f^{n}\right|_{E^{1}(x)} .
$$

In $E^{1}\left(f^{n} x\right)$ we denote $0_{n}=0_{f^{n} x}$ and $\bar{y}_{n}=d f_{x}^{n}(\bar{y})=\left(h_{f_{x}^{n}}\right)^{-1}\left(f^{n} y\right)$. Then

and

$$
\begin{aligned}
d \bar{H}(\bar{y}) & =\left.\left(\left.d f^{n}\right|_{E^{1}(z)}\right)^{-1} \circ d \bar{H}_{n}\left(\bar{y}_{n}\right) \circ d f^{n}\right|_{E^{1}(x)} \\
d \bar{H}\left(0_{x}\right) & =\left.\left(\left.d f^{n}\right|_{E^{1}(z)}\right)^{-1} \circ d \bar{H}_{n}\left(0_{n}\right) \circ d f^{n}\right|_{E^{1}(x)} .
\end{aligned}
$$

We estimate the norm of the difference between $d \bar{H}(\bar{y})$ and $d \bar{H}\left(0_{x}\right)$ as follows.

$$
\begin{aligned}
\left\|d \bar{H}(\bar{y})-d \bar{H}\left(0_{x}\right)\right\| & =\left\|\left.\left(\left.d f^{n}\right|_{E^{1}(z)}\right)^{-1} \circ\left(d \bar{H}_{n}\left(\bar{y}_{n}\right)-d \bar{H}_{n}\left(0_{n}\right)\right) \circ d f^{n}\right|_{E^{1}(x)}\right\| \\
& \leq\left\|\left(\left.d f^{n}\right|_{E^{1}(z)}\right)^{-1}\right\| \cdot\left\|d \bar{H}_{n}\left(\bar{y}_{n}\right)-d \bar{H}_{n}\left(0_{n}\right)\right\| \cdot\left\|\left.d f^{n}\right|_{E^{1}(x)}\right\|
\end{aligned}
$$

Since the holonomy maps are $C^{1, \beta}$ with uniform Hölder constant, so are $\bar{H}$. Hence

$$
\left\|d \bar{H}_{n}\left(\bar{y}_{n}\right)-d \bar{H}_{n}\left(0_{n}\right)\right\| \leq K \cdot\left(\operatorname{dist}\left(\bar{y}_{n}, 0_{n}\right)\right)^{\beta} \leq K \cdot\left(\operatorname{dist}\left(\bar{y}, 0_{x}\right)\right)^{\beta} \cdot\left\|\left.d f^{n}\right|_{E^{1}(x)}\right\|^{\beta} .
$$

Therefore

$\left\|d \bar{H}(\bar{y})-d \bar{H}\left(0_{x}\right)\right\| \leq K \cdot\left(\operatorname{dist}\left(\bar{y}, 0_{x}\right)\right)^{\beta} \cdot\left\|\left(\left.d f^{n}\right|_{E^{1}(z)}\right)^{-1}\right\| \cdot\left\|\left.d f^{n}\right|_{E^{1}(x)}\right\|^{1+\beta} \rightarrow 0$ as $n \rightarrow \infty$. This implies that $d \bar{H}(\bar{y})=d \bar{H}\left(0_{x}\right)$ for any $\bar{y}$ in $E^{1}(x)$. This shows that $d \bar{H}$ is constant on $E^{1}(x)$, and therefore the map $\bar{H}$ is linear.

Since $h_{x}$ and $h_{z}$ are $C^{\infty}$ diffeomorphisms, the holonomy map $H=\left(h_{z}\right)^{-1} \circ \bar{H} \circ$ $h_{x}: W^{1}(x) \rightarrow W^{1}(z)$ is also a $C^{\infty}$ diffeomorphism. Recall that the linearization depends continuously on the base point in $C^{\infty}$ topology. Then, since $\bar{H}$ is linear and depends continuously on $x$ and $z$, the holonomy map $H=H_{x z}$ depends continuously on $x$ and $z$ in $C^{\infty}$ topology. This implies that $E^{1}$ is uniformly $C^{\infty}$ along the leaves of $W[20]$. 
Corollary 4.8 Under the assumptions of Theorem 3.1, all coarse Lyapunov distributions $W^{i}$ are $C^{\infty}$ on $\mathcal{M}$. Also, the holonomy maps between the leaves of $W^{i}$ along the leaves of $W^{j}$ are affine.

Proof We fix coarse Lyapunov foliations $W^{i}$ and $W^{j}$. To apply Proposition 4.7 we consider an element $f$ of the action which contracts $E^{i} \oplus E^{j}$. Such an element exists since the action is TNS, and it is uniformly quasiconformal on $E^{i}$ by assumption (2) of the theorem. In Section 4.3 we showed that $E^{j}$ is $C^{1, \beta}$ along the leaves of $W=W^{i} \oplus W^{j}$ with some $\beta>0$ and a uniform Hölder constant on $\mathcal{M}$. Hence so are the holonomy maps $H$ along $W^{j}$ [20]. By Lemma 4.10, $\left\|\left(\left.d f^{n}\right|_{E^{i}(z)}\right)^{-1}\right\| \cdot\left\|\left.d f^{n}\right|_{E^{i}(x)}\right\|$ is bounded by a constant independent of $n$, and hence

$$
\left\|\left(\left.d f^{n}\right|_{E^{i}(z)}\right)^{-1}\right\| \cdot\left\|\left.d f^{n}\right|_{E^{i}(x)}\right\|^{1+\beta} \rightarrow 0
$$

for any $\beta>0$. Now it follows from Proposition 4.7 that the holonomy maps are affine and uniformly $C^{\infty}$, and $E^{j}$ is uniformly $C^{\infty}$ along the leaves of $W$.

In particular, we have established that any coarse Lyapunov distribution is uniformly $C^{\infty}$ along the leaves of any coarse Lyapunov foliation. To conclude that any coarse Lyapunov distribution is $C^{\infty}$ on $\mathcal{M}$ we use the following well-known result (see Katok and Lewis [12] and Goetze and Spatzier [6]), which is obtained by inductive application of Journé Lemma 4.2.

Lemma 4.9 Let $\phi$ be a map from $\mathcal{M}$ to a finite-dimensional $C^{\infty}$ manifold. If $\phi$ is uniformly $C^{\infty}$ along the leaves of every coarse Lyapunov foliation then it is $C^{\infty}$ on $\mathcal{M}$.

To complete the proof of Corollary 4.8, it remains to establish the following lemma.

Lemma 4.10 Let $E$ be a Hölder continuous distribution invariant under a diffeomorphism $f$. Suppose that $f$ is uniformly quasiconformal on $E$. Let $x$ and $z$ be two points such that for some $K_{1}$ and $0<\lambda<1$, dist $\left(f^{n} x, f^{n} z\right) \leq K_{1} \cdot \lambda^{n}$ for all $n \geq 0$. Then there exists a constant $K$ such that for all $n \geq 0$,

$$
\left\|\left(\left.d f^{n}\right|_{E(z)}\right)^{-1}\right\| \cdot\left\|\left.d f^{n}\right|_{E(x)}\right\| \leq K .
$$

Note that the estimate (4-4) implies the one in Lemma 4.5, and in the uniformly quasiconformal case the two estimates are, in fact, equivalent. The main difference is that in Lemma 4.10 the points $x$ and $z$ are not required to be on the same leaf of a foliation tangential to $E$. Hence the proof is different and requires a stronger assumption. 
Proof Since $f$ is uniformly quasiconformal on $E$, Theorem 2.7 implies that it is conformal with respect to a Hölder continuous Riemannian metric on $E$. We will use this metric in the proof. Note that (4.4) is independent of the choice of a continuous metric on $E$.

To simplify the notations, in this proof only we write $d f_{x}^{i}$ instead of $\left.d f^{i}\right|_{E(x)}$. Since $E$ is Hölder continuous, $\left.d f\right|_{E}$ is also Hölder continuous with some exponent $\beta>0$. Thus we obtain

$$
\frac{\left\|d f_{x}\right\|}{\left\|d f_{z}\right\|} \leq 1+\frac{\left|\left\|d f_{x}\right\|-\left\|d f_{z}\right\|\right|}{\left\|d f_{z}\right\|} \leq 1+\frac{K_{2} \cdot(\operatorname{dist}(x, z))^{\beta}}{\min _{z}\left\|d f_{z}\right\|}=1+K_{3} \cdot(\operatorname{dist}(x, z))^{\beta} .
$$

Since $f$ is conformal on $E,\left\|\left(d f_{z}\right)^{-1}\right\|=\left\|d f_{z}\right\|^{-1}$, and we can estimate

$$
\begin{aligned}
\left\|\left(d f_{z}^{n}\right)^{-1}\right\| \cdot\left\|d f_{x}^{n}\right\| \\
\leq\left\|\left(d f_{z}\right)^{-1}\right\| \cdot\left\|\left(d f_{f z}\right)^{-1}\right\| \ldots\left\|\left(d f_{f^{n-1} z}\right)^{-1}\right\| \cdot\left\|d f_{x}\right\| \cdot\left\|d f_{f x}\right\| \ldots\left\|d f_{f^{n-1} x}\right\| \\
\quad=\frac{\left\|d f_{x}\right\|}{\left\|d f_{z}\right\|} \cdot \frac{\left\|d f_{f x}\right\|}{\left\|d f_{f_{z}}\right\|} \cdots \frac{\left\|d f_{f^{n-1} x}\right\|}{\left\|d f_{f^{n-1} z}\right\|} \leq \prod_{i=0}^{n-1}\left(1+K_{3} \cdot\left(\operatorname{dist}\left(f^{i} x, f^{i} z\right)\right)^{\beta}\right) \\
\quad \leq \prod_{i=0}^{n-1}\left(1+K_{3} \cdot\left(K_{1} \cdot \lambda^{i}\right)^{\beta}\right) \leq \prod_{i=0}^{n-1}\left(1+K_{4} \cdot \lambda^{i \beta}\right) \leq K .
\end{aligned}
$$

\subsection{The linearizations depend smoothly on the basepoint}

Let $W^{1}$ and $W^{2}$ be two coarse Lyapunov foliations and let $W=W^{1} \oplus W^{2}$ be their sum. In Section 4.5 we established that the holonomy maps between the leaves of $W^{1}$ along $W^{2}$ are affine. We will use this fact to show that the linearizations $h_{x}^{1}: W^{1}(x) \rightarrow E^{1}(x)$ depend smoothly on the base point along the leaves of $W$.

Proposition 4.11 If $W^{1}$ and $W^{2}$ are two coarse Lyapunov foliations then the linearizations $h_{x}^{1}$ depend uniformly $C^{\infty}$ on the base point along the leaves of $W^{1} \oplus W^{2}$.

Proof First we construct a local linearization $h$ along $W$ using linearizations $h^{1}$ along $W^{1}$ and $h^{2}$ along $W^{2}$. Let $E$ be the distribution tangent to $W$ and let $U$ be a small open neighborhood of a point $x$ in $W$. We define the map $h_{x}: U \rightarrow E(x)$ as follows:

$$
\left.h_{x}\right|_{W^{1}(x)}=h_{x}^{1},\left.\quad h_{x}\right|_{W^{2}(x)}=h_{x}^{2},
$$

and for a point $p$ in $U$ we set

$$
h_{x}(p)=h_{x}^{1}(y)+h_{x}^{2}(z) \text {, where } y=W^{2}(p) \cap W^{1}(x), z=W^{1}(p) \cap W^{2}(x) .
$$


Since the foliations $W^{1}$ and $W^{2}$ are $C^{\infty}$ smooth along the leaves of $W$, the map $h_{x}$ is a $C^{\infty}$ diffeomorphism. It is easy to see that the family of maps $h_{x}$ satisfies conditions (1), (2), and (3) of Proposition 4.4 and thus $h$ gives a local nonstationary linearization along $W$. However, we do not use properties (1) and (2) in the proof.

Let us identify $E(x)$ with $\mathbb{R}^{n} \times \mathbb{R}^{m}$ in such a way that $E^{1}(x)$ corresponds to $\mathbb{R}^{n}$, and $E^{2}(x)$ corresponds to $\mathbb{R}^{m}$. Then $h_{x}$ identifies the neighborhood $U \subset W$ of $x$ with an open neighborhood $\bar{U}$ of 0 in $\mathbb{R}^{n} \times \mathbb{R}^{m}$. It is clear from the construction of $h_{x}$ that the leaves of $W^{1}$ and $W^{2}$ correspond to subspaces parallel to $\mathbb{R}^{n}$ and $\mathbb{R}^{m}$ respectively. For a point $p$ in $U$, the tangent space $E_{p}$ is identified with $\mathbb{R}^{n} \times \mathbb{R}^{m}$ by $\left(d h_{x}\right)_{p}$ in such a way that $0_{p}$ corresponds to $\bar{p}=h_{x}(p)$. We will show that the maps $h_{p}^{1}, p \in U$, are identity maps when written in these local coordinates. In other words, this coordinate system coincides with the linearization on every leaf of $W^{1}$. This implies that the linearizations $h_{p}^{1}$ depend smoothly on $p$.

Let $\psi_{p}$ be the restriction of $h_{x}$ to $W^{1}(p)$. By the construction of $h_{x}$, the diffeomorphism $\psi_{p}$ can be expressed as

$$
\psi_{p}=\bar{H}_{0, \bar{p}} \circ h_{x}^{1} \circ H_{p, x},
$$

where $H_{p, x}$ is the holonomy map from $W^{1}(p)$ to $W^{1}(x)$ along the leaves of $W^{2}$, and $\bar{H}_{0, \bar{p}}$ is the projection from $\mathbb{R}^{n}$ to $\mathbb{R}_{\bar{p}}^{n}=\mathbb{R}^{n}+\bar{p}$ along $\mathbb{R}^{m}$ in $\mathbb{R}^{n} \times \mathbb{R}^{m}$. Since the holonomy map $H_{p, x}$ is affine by Corollary 4.8, the map $\psi_{p}$ is also affine.

We denote by $\bar{h}_{p}^{1}$ the coordinate representation of $h_{p}^{1}$, ie,

$$
\bar{h}_{p}^{1}=d \psi_{p}(p) \circ h_{p}^{1} \circ \psi_{p}^{-1}: \mathbb{R}_{\bar{p}}^{n} \cap U \rightarrow \mathbb{R}_{\bar{p}}^{n} .
$$

Since $\psi_{p}$ is affine, we conclude that $\bar{h}_{p}^{1}$ is also affine. We note that

$$
\bar{h}_{p}^{1}(\bar{p})=\bar{p} \text { and } d \bar{h}_{p}^{1}(\bar{p})=d \psi_{p}(p) \circ d h_{p}^{1}(p) \circ d\left(\psi_{p}^{-1}\right)(\bar{p})=\mathrm{Id},
$$

since $d h_{p}^{1}(p)=\mathrm{Id}$ by Proposition 4.4(2). Hence the affine map $\bar{h}_{p}^{1}$ is the identity map, and therefore the maps $h_{p}^{1}$ depend uniformly $C^{\infty}$ on $p$. The uniformity comes from the fact that $h_{x}$ depends continuously on $x$ in $C^{\infty}$ topology.

\subsection{Smooth affine connection}

In this section we construct an $\alpha$-invariant $C^{\infty}$ affine connection on $\mathcal{M}$. We say that an affine connection $\nabla$ is of class $C^{r}, r \geq 0$, if $\nabla_{X} Y$ is a $C^{r}$ vector field for any two $C^{\infty}$ vector fields $X$ and $Y$.

It was proved by Feres in [3] that an Anosov diffeomorphism which is 1/2-pinched on its stable and unstable distributions preserves a unique continuous affine connection. In 
fact, such a connection exists on any invariant distribution where a diffeomorphism is a 1/2-pinched contraction [4]. The connections on the stable and unstable distributions can be combined into a connection on the manifold. However, the connection on the (un)stable distribution is not known to be smooth transversally even if the distribution is smooth. Feres also noted in [4] that the exponential map of an invariant connection gives a nonstationary local linearization.

We consider coarse Lyapunov distributions and use the nonstationary linearizations to obtain invariant affine connections on them. Their smoothness will follow from the smoothness of the linearizations. It can be seen from Proposition 4.6 that these affine connections are the same as the ones defined by the affine structures on the leaves of coarse Lyapunov foliations.

First we define an $\alpha$-invariant affine connection $\nabla^{i}$ along each coarse Lyapunov foliation $W^{i}$. At each point $x$ in $\mathcal{M}$, pull back the affine connection $\bar{\nabla}^{E^{i}(x)}$ on the tangent space $E^{i}(x)$ using the map $h_{x}^{i}: W^{i}(x) \rightarrow E^{i}(x)$ to define an $\alpha$-invariant affine connection $\nabla^{i}$ along each coarse Lyapunov foliation $W^{i}$. More precisely, for vector fields $X^{i}$ and $Y^{i}$ on $W^{i}$ we define

$$
\left(\nabla_{X^{i}}^{i} Y^{i}\right)(x)=\left(h_{x}^{i}\right)_{*}^{-1}\left(\bar{\nabla}_{\bar{X}^{i}}^{E^{i}(x)} \bar{Y}^{i}\right)
$$

where $\bar{X}^{i}=\left(h_{x}^{i}\right)_{*} X^{i}$ and $\bar{Y}^{i}=\left(h_{x}^{i}\right)_{*} Y^{i}$ are the push-forwards of $X^{i}$ and $Y^{i}$. It is easy to see that $\nabla^{i}$ is an $\alpha$-invariant affine connection, which is as smooth as the dependence of $h_{x}^{i}$ on $x$. Thus, by Proposition 4.11, $\nabla^{i}$ is uniformly $C^{\infty}$ along the leaves of any coarse Lyapunov foliation. It follows from Lemma 4.9 that $\nabla^{i}$ is $C^{\infty}$ on $\mathcal{M}$.

Now we define an affine connection $\nabla$ on $\mathcal{M}$ using a standard construction. Let $X$ and $Y$ be two vector fields on $\mathcal{M}$. We decompose $X=\sum X^{i}$ and $Y=\sum Y^{i}$, where $X^{i}, Y^{i} \in E^{i}$. Then

$$
\nabla_{X} Y=\sum \nabla_{X^{i}}^{i} Y^{i}+\sum_{i \neq j} \Pi_{j}\left[X^{i}, Y^{j}\right],
$$

where $\Pi_{j}$ is the projection onto $E^{j}$, defines an affine connection. Since the distributions $E^{i}$ and the connections $\nabla^{i}$ are $C^{\infty}$, so is $\nabla$. Since $\nabla^{i}$ and $E^{i}$ are $\alpha$-invariant, so is $\nabla$. Thus we constructed an $\alpha$-invariant $C^{\infty}$ affine connection on $\mathcal{M}$.

\subsection{Smooth conjugacy to a toral action}

In this section we complete the proof of Theorem 3.1. First we show that the action is $C^{\infty}$-conjugate to an action by affine automorphisms of an infranilmanifold. 
Let $f$ be a transitive Anosov element of the action $\alpha$. Then $f$ preserves the $C^{\infty}$ affine connection $\nabla$ constructed in Section 4.7. Also, its stable and unstable distributions are $C^{\infty}$ as direct sums of $C^{\infty}$ coarse Lyapunov distributions. It follows from the main result of $\mathrm{Y}$ Benoist and $\mathrm{F}$ Labourie in [1] that $f$ is conjugate to an automorphism of an infranilmanifold $\mathcal{N}$ by a $C^{\infty}$ diffeomorphism $\phi$. It is known that any diffeomorphism commuting with an Anosov automorphism of an infranilmanifold is an affine automorphism itself (see Section 2.3). Hence $\phi$ conjugates $\alpha$ to an action $\bar{\alpha}$ by affine automorphisms of $\mathcal{N}$.

Now we complete the proof by showing that $\mathcal{N}$ is finitely covered by a torus. We use joint integrability of the coarse Lyapunov foliations and uniform quasiconformality of the action on them.

The conjugacy maps the (un)stable manifolds for elements of $\alpha$ to the (un)stable manifolds for elements of $\bar{\alpha}$, and the course Lyapunov foliations of $\alpha$ are mapped to the course Lyapunov foliations of $\bar{\alpha}$. It follows that any two course Lyapunov foliations of $\bar{\alpha}$ are jointly integrable. Since the conjugacy is smooth, the Lyapunov functionals of the two actions coincide, and $\bar{\alpha}$ is uniformly quasiconformal on its course Lyapunov distributions. Also, the action $\bar{\alpha}$ is TNS, and hence it has no negatively proportional Lyapunov functionals.

The infranilmanifold $\mathcal{N}$ is finitely covered by a nilmanifold $N / \Gamma$, where $N$ is a simply connected nilpotent Lie group, and $\Gamma$ is a cocompact lattice in $N$. We need to show that $N$ is abelian. The Lie algebra $\mathfrak{n}$ of $N$ splits into Lyapunov subspaces $\mathfrak{e}_{i}$ with Lyapunov functionals $\chi_{i}$ of the action $\bar{\alpha}$. If for $u \in \mathfrak{e}_{i}$ and $v \in \mathfrak{e}_{j}$ the bracket $[u, v]$ is nonzero, then $[u, v]$ belongs to a Lyapunov subspace with Lyapunov functional $\chi_{i}+\chi_{j}$. We recall that a coarse Lyapunov subspace is a direct sum of Lyapunov subspaces corresponding to positively proportional Lyapunov functionals.

Suppose that $[u, v] \neq 0$ for some $u$ and $v$ which belong to two different coarse Lyapunov subspaces. Then $\chi_{i}$ and $\chi_{j}$ are not positively proportional and hence are not proportional. If follows that $\chi_{i}+\chi_{j}$ is not proportional to either one of them. Hence $[u, v]$ belongs to a coarse Lyapunov subspace different from the ones containing $u$ and $v$. This contradicts the fact that any two course Lyapunov foliations of $\bar{\alpha}$ are jointly integrable.

Suppose that $u$ and $v$ are in the same coarse Lyapunov subspace $\mathfrak{e}$. Uniform quasiconformality of the action on $\mathfrak{e}$ implies that all vectors in $\mathfrak{e}$ are expanded/contracted at the same rate, ie, there is only one Lyapunov functional $\chi$ corresponding to $\mathfrak{e}$. If $[u, v]$ is nonzero, then it belongs to a Lyapunov subspace with Lyapunov functional $2 \chi$. But this subspace must be contained in $\mathfrak{e}$, which is impossible. 
We conclude that $[u, v]=0$ for any $u$ and $v$ in the Lie algebra $\mathfrak{n}$. Thus the Lie group $N$ is abelian, and the infranilmanifold $\mathcal{N}$ is finitely covered by a torus. This completes the proof of Theorem 3.1.

\section{Proof of Theorem 3.4 and its corollaries}

\subsection{Proof of Theorem 3.4}

The proof of this theorem follows the same steps as the proof of Theorem 3.1. Below we describe the necessary adjustments.

The coarse Lyapunov distributions are substituted by the distributions $E^{i}$ given in the theorem. The fact that any two such distributions are contracted by some element of the action is given by condition (2) of the theorem. Since these distributions are intersections of some stable distributions, they have most of the properties of coarse Lyapunov distributions. In particular, their integral foliations are Hölder continuous with uniformly $C^{\infty}$ leaves.

Let $E^{1}$ and $E^{2}$ be two distributions, and let $W^{1}$ and $W^{2}$ be the corresponding foliations. As in Section 4.2, we show that the leaves of the foliation $W=W^{1} \oplus W^{2}$, given by assumption (1), are smooth. Then we show the smoothness of $E^{1}$ along the leaves of $W$ using the following proposition in place of Proposition 4.3.

Proposition 5.1 Suppose that there exists an element $f$ that expands $E^{1}$ faster than it expands $E^{2}$. Then $E^{1}$ is $C^{\infty}$ along the leaves of $W$, ie, its derivatives of all orders in $W$ directions exist, are continuous along the leaves of $W$ and uniformly bounded on $\mathcal{M}$.

Proof We apply the $C^{r}$ Section Theorem as in the proof of Proposition 4.3. The assumptions on $f$ directly imply that $\sup _{x \in M} k_{x}<1$, ie, the induced map $F$ contracts the fibers. Moreover, since $f$ expands $W$, we see that $\alpha_{x}<1$, and thus

$$
\sup _{x \in M} k_{x} \alpha_{x}^{r}<1 \text { for any } r>0 .
$$

The $C^{r}$ Section Theorem implies that the distribution $E^{1}$ is $C^{\infty}$ smooth along the leaves of $W$.

As in Section 4.4, we obtain nonstationary linearizations of the action along the leaves of foliations $W^{i}$ and the associated affine structures. In this case, (4-2) follows from the 1/2-pinching assumption. 
It follows from condition (3) of the theorem and Proposition 5.1 that the holonomies of $W^{1}$ between the leaves of $W^{2}$ are $C^{\infty}$ along the leaves of $W=W^{1} \oplus W^{2}$ and vise versa. Also, the 1/2-pinching assumption implies (4-3) with $\beta=1$. Hence we can apply Proposition 4.7 to show that the holonomies are affine and uniformly $C^{\infty}$ along the leaves of $W$. As in Section 4.5, it follows that all distributions $W^{i}$ are $C^{\infty}$ on $\mathcal{M}$.

As in Sections 4.6 and 4.7, we show that the nonstationary linearizations depend $C^{\infty}$ on the base point and construct a $C^{\infty}$-invariant affine connection. As in the beginning of Section 4.8, we conclude that the action is $C^{\infty}$-conjugate to an action by affine automorphisms of an infranilmanifold.

\subsection{Proof of Corollary 3.7}

We need to show that the infranilmanifold obtained in Theorem 3.4 is finitely covered by a torus. The argument is similar to the one in Section 4.8. We use joint integrability of the coarse Lyapunov foliations and the 1/2-pinching. The only difference is in showing that $[u, v]=0$ for any $u$ and $v$ which belong to the same coarse Lyapunov subspace $\mathfrak{e}$. Suppose that $[u, v] \neq 0$ for some $u$ and $v$ in $\mathfrak{e}$. Then the corresponding Lyapunov functionals $\chi_{i}$ and $\chi_{j}$ are positively proportional. Hence they are positively proportional to $\chi_{i}+\chi_{j}$, and the Lyapunov subspace of $\chi_{i}+\chi_{j}$ is also contained in $\mathfrak{e}$. It is easy to see that this contradicts the existence of an element which is 1/2-pinched on $\mathfrak{e}$.

\subsection{Proof of Corollary 3.9}

It suffices to verify assumptions (2) and (3) of Theorem 3.4. Let $E^{1}$ and $E^{2}$ be two coarse Lyapunov distributions. Let $\chi_{\min }^{1}$ and $\chi_{\min }^{2}$ be the corresponding functionals, and let $\mathcal{H}^{1}$ and $\mathcal{H}^{2}$ be their negative half-spaces in $\mathbb{R}^{k}$. If follows from assumption (2) of Corollary 3.9 that the negative half-space of $\chi_{\max }^{i}$ is $\mathcal{H}^{i}$, and that all elements in $\mathcal{H}^{i}$ contract $E^{i}$.

Since the action is TNS Anosov, there exists an element which contracts both $E^{1}$ and $E^{2}$. Hence the intersection $\mathcal{K}=\mathcal{H}^{1} \cap \mathcal{H}^{2}$ is a nonempty open convex cone in $\mathbb{R}^{k}$. It follows from condition (2) of the corollary that all elements in $\mathcal{K}$ contract both $E^{1}$ and $E^{2}$ and are 1/2-pinched on them. This verifies assumption (2) of Theorem 3.4. To verify assumption (3) of the Theorem, we take an element $a$ in $\mathbb{Z}^{k} \cap(-\mathcal{K})$ close to the boundary of $\mathcal{H}^{2}$ and away from the boundary of $\mathcal{H}^{1}$. Clearly, such an element expands $E^{1}$ faster than $E^{2}$. This completes the proof of the corollary. 


\subsection{Proof of Corollary 3.8}

It is well known that an Anosov $\mathbb{Z}^{k}$ action by toral automorphisms is topologically conjugate to a $C^{1}$-small perturbation. To show the smoothness of the conjugacy we verify the assumptions of Theorem 3.4 for the perturbed action $\widetilde{\alpha}$.

First we consider the unperturbed action $\alpha$ by toral automorphisms. For any coarse Lyapunov distribution $E$ we denote by $\chi_{\min }$ and $\chi_{\max }$ the minimal and the maximal of the Lyapunov functionals corresponding to $E$. The existence of a regular element which is $1 / 2$-pinched on $E$ implies that condition (2) of Corollary 3.9 is satisfied. Hence we can verify assumptions (2) and (3) of Theorem 3.4 for $\alpha$ as in Section 5.3.

Now we consider a $C^{1}$-small perturbation $\widetilde{\alpha}$. For any coarse Lyapunov distribution $E$ of $\alpha$ there exists a corresponding $\widetilde{\alpha}$-invariant distribution $\widetilde{E}$ close to $E$. Each $\widetilde{E}$ is the intersection of the corresponding stable distributions for $\widetilde{\alpha}$, and the tangent bundle is the direct sum of the distributions $\widetilde{E}$. If the perturbation is sufficiently $C^{1}$-small, the assumptions (2) and (3) of Theorem 3.4 are satisfied for the distributions $\widetilde{E}$. Assumption (1) follows from the existence of topological conjugacy to $\alpha$. Now Theorem 3.4 implies that the conjugacy is $C^{\infty}$.

\section{References}

[1] Y Benoist, F Labourie, Sur les difféomorphismes d'Anosov affines à feuilletages stable et instable différentiables, Invent. Math. 111 (1993) 285-308 MR1198811

[2] M Einsiedler, T T Fisher, Differentiable rigidity for hyperbolic toral actions, to appear in Israel J. Math.

[3] R Feres, The invariant connection of a $\frac{1}{2}$-pinched Anosov diffeomorphism and rigidity, Pacific J. Math. 171 (1995) 139-155 MR1362981

[4] R Feres, A differential-geometric view of normal forms of contractions, from: "Modern dynamical systems and applications", Cambridge Univ. Press, Cambridge (2004) 103121 MR2090767

[5] J Franks, Anosov diffeomorphisms on tori, Trans. Amer. Math. Soc. 145 (1969) 117124 MR0253352

[6] E R Goetze, R J Spatzier, Smooth classification of Cartan actions of higher rank semisimple Lie groups and their lattices, Ann. of Math. (2) 150 (1999) 743-773 MR1740993

[7] MW Hirsch, C C Pugh, M Shub, Invariant manifolds, Springer, Berlin (1977) MR0501173

[8] S Hurder, Rigidity for Anosov actions of higher rank lattices, Ann. of Math. (2) 135 (1992) 361-410 MR1154597 
[9] J-L Journé, A regularity lemma for functions of several variables, Rev. Mat. Iberoamericana 4 (1988) 187-193 MR1028737

[10] B Kalinin, A Katok, Invariant measures for actions of higher rank abelian groups, from: "Smooth ergodic theory and its applications (Seattle, WA, 1999)", Proc. Sympos. Pure Math. 69, Amer. Math. Soc., Providence, RI (2001) 593-637 MR1858547

[11] B Kalinin, R Spatzier, On the classification of Cartan actions, to appear in Geom. Funct. Anal.

[12] A Katok, J Lewis, Local rigidity for certain groups of toral automorphisms, Israel J. Math. 75 (1991) 203-241 MR1164591

[13] A Katok, V Niţică, A Török, Non-abelian cohomology of abelian Anosov actions, Ergodic Theory Dynam. Systems 20 (2000) 259-288 MR1747022

[14] A Katok, R J Spatzier, Differential rigidity of Anosov actions of higher rank abelian groups and algebraic lattice actions, Tr. Mat. Inst. Steklova 216 (1997) 292-319 MR1632177

[15] E Lindenstrauss, Rigidity of multiparameter actions, Israel J. Math. 149 (2005) 199226 MR2191215

[16] A Manning, There are no new Anosov diffeomorphisms on tori, Amer. J. Math. 96 (1974) 422-429 MR0358865

[17] S E Newhouse, On codimension one Anosov diffeomorphisms, Amer. J. Math. 92 (1970) 761-770 MR0277004

[18] V Niţică, A Török, On the cohomology of Anosov actions, from: "Rigidity in dynamics and geometry (Cambridge, 2000)", Springer, Berlin (2002) 345-361 MR1919410

[19] J Palis, J-C Yoccoz, Centralizers of Anosov diffeomorphisms on tori, Ann. Sci. École Norm. Sup. (4) 22 (1989) 99-108 MR985856

[20] C Pugh, M Shub, A Wilkinson, Hölder foliations, Duke Math. J. 86 (1997) 517-546 MR1432307

[21] V Sadovskaya, On uniformly quasiconformal Anosov systems, Math. Res. Lett. 12 (2005) 425-441 MR2150895

Department of Mathematics and Statistics, University of South Alabama

Mobile, AL 36688, USA

kalinin@jaguar1.usouthal.edu, sadovska@jaguar1.usouthal.edu

Proposed: Benson Farb

Seconded: David Gabai, Leonid Polterovich
Received: 8 September 2005

Revised: 11 June 2006 